\title{
Singular values and fixed points of family of generating function of Bernoulli's numbers
}

\author{
Mohammad Sajid* \\ College of Engineering, Qassim University, Buraidah, Al-Qassim, Saudi Arabia. \\ Communicated by R. Saadati
}

\begin{abstract}
Singular values and fixed points of one parameter family of generating function of Bernoulli's numbers, $g_{\lambda}(z)=\lambda \frac{z}{e^{z}-1}, \lambda \in \mathbb{R} \backslash\{0\}$, are investigated. It is shown that the function $g_{\lambda}(z)$ has infinitely many singular values and its critical values lie outside the open disk centered at origin and having radius $\lambda$. Further, the real fixed points of $g_{\lambda}(z)$ and their nature are determined. The results found are compared with the functions $\lambda \tan z, E_{\lambda}(z)=\lambda \frac{e^{z}-1}{z}$ and $f_{\lambda}(z)=\lambda \frac{z}{z+4} e^{z}$ for $\lambda>0$. (C)2015 All rights reserved.
\end{abstract}

Keywords: Fixed points, critical values, singular values.

2010 MSC: 30D05, 37C25, 58K05.

\section{Introduction}

The singular values and fixed points play a vital role in the dynamics or iteration of meromorphic functions, i.e. the functions whose only singularities in the complex plane are poles. The theory of dynamics or iteration of functions can be seen in [1, 3, 6]. The investigation of dynamics of meromorphic functions with infinitely many bounded or unbounded singular values is scarcely found in comparison to that of functions with finitely many singular values. To fill this gap, the present work describes some dynamical properties of the family of transcendental meromorphic functions. The dynamics of entire functions $\lambda \frac{\sinh (z)}{z}$ and $\lambda \frac{e^{z}-1}{z}, \lambda>0$ with infinitely many bounded singular values are investigated by Prasad [8], and Kapoor and Prasad [5] respectively. Further, the dynamics of certain class of critically bounded entire transcendental functions is studied by Prasad and Nayak [9. Some properties of bounded-type entire functions are shown in [10]. The dynamics of transcendental meromorphic functions $\lambda \frac{\sinh ^{2}(z)}{z^{4}}$ with infinitely many bounded

\footnotetext{
*Corresponding author

Email address: msajid@qec.edu.sa (Mohammad Sajid)
} 
singular values was found in [11] and the dynamics of certain transcendental meromorphic functions with unbounded singular values was explored by Nayak and Prasad [7]. The singular values of transcendental meromorphic functions were also discussed by Zheng [13].

The motivation of the present work comes from the fact that the function $\frac{z}{e^{z}-1}$ is a generating function of the Bernoulli's numbers. These Bernoulli's numbers $B_{0}=1, B_{1}=-\frac{1}{2}, B_{2}=\frac{1}{6}, B_{3}=0, B_{4}=-\frac{1}{30}$, $B_{5}=0, B_{6}=\frac{1}{42}, \ldots$ are coefficients in the series expansion $\frac{z}{e^{z}-1}=\sum_{k=0}^{\infty} B_{k} z^{k}$ for $|z|<2 \pi$ and also holds strong association with Riemann zeta function [4]. By considering one parameter family of function $\lambda \frac{z}{e^{z}-1}$, we have to determine its singular values and fixed points.

Let,

$$
\mathcal{G}=\left\{g_{\lambda}(z)=\lambda \frac{z}{e^{z}-1} \text { and } g_{\lambda}(0)=\lambda: \lambda \in \mathbb{R} \backslash\{0\}, z \in \mathbb{C}\right\}
$$

be one parameter family of transcendental meromorphic functions. The function $g_{\lambda} \in \mathcal{G}$ is a transcendental meromorphic function with infinitely many poles at $\{2 \pi k i: k \in \mathbb{Z}\}$. Further, all poles are simple and all are lying on imaginary axis. Moreover, the function $g_{\lambda}(z)$ is neither even nor odd and not periodic.

The following are the basic definitions which are needed in the sequel: A point $z^{*}$ is said to be a critical point of $f(z)$ if $f^{\prime}\left(z^{*}\right)=0$. The value $f\left(z^{*}\right)$ corresponding to a critical point $z^{*}$ is called a critical value of $f(z)$. A point $w \in \hat{\mathbb{C}}=\mathbb{C} \cup\{\infty\}$ is said to be an asymptotic value for $f(z)$, if there exists a continuous curve $\gamma:[0, \infty) \rightarrow \hat{\mathbb{C}}$ satisfying $\lim _{t \rightarrow \infty} \gamma(t)=\infty$ and $\lim _{t \rightarrow \infty} f(\gamma(t))=w$. A singular value of $f$ is defined to be either a critical value or an asymptotic value of $f$. A function $f$ is called critically bounded or functions of bounded type if the set of all singular values of $f$ is bounded, otherwise unbounded-type. A point $z$ is said to be a fixed point of function $f(z)$ if $f(z)=z$. A fixed point $z_{0}$ is called an attracting, neutral (indifferent) or repelling if $\left|f^{\prime}\left(z_{0}\right)\right|<1,\left|f^{\prime}\left(z_{0}\right)\right|=1$ or $\left|f^{\prime}\left(z_{0}\right)\right|>1$ respectively.

The following is the organization of this paper: In Section 2, it is found that the function $g_{\lambda} \in \mathcal{G}$ has infinitely many singular values. Further, it is shown that all the critical values of $g_{\lambda}(z)$ lie outside the open disk centered at origin and having radius $\lambda$. The real fixed points of $g_{\lambda} \in \mathcal{G}$ and their nature are determined in Section 3. Finally, the results found here are compared with the analogous results for the functions $\lambda \tan z, E_{\lambda}(z)=\lambda \frac{e^{z}-1}{z}$ and $f_{\lambda}(z)=\lambda \frac{z}{z+4} e^{z}$ for $\lambda>0$.

\section{Singular Values of $g_{\lambda} \in \mathcal{G}$}

In this section, it is proved that the function $g_{\lambda} \in \mathcal{G}$ has infinitely many singular values and all the critical values of $g_{\lambda}(z)$ lie in the left half plane outside the disk centered at origin and having radius $\lambda$.

The following theorem gives the function $g_{\lambda}(z)$ has infinitely many singular values:

Theorem 2.1. Let $g_{\lambda} \in \mathcal{G}$. Then, the function $g_{\lambda}(z)$ possesses infinitely many singular values.

Proof. For critical points, $g_{\lambda}^{\prime}(z)=\lambda \frac{(1-z) e^{z}-1}{\left(e^{z}-1\right)^{2}}=0$. This gives the equation $(z-1) e^{z}+1=0$. The real and imaginary parts of this equation are

$$
\begin{gathered}
\frac{y}{\sin y}-e^{y \cot y-1}=0 \\
x=1-y \cot y
\end{gathered}
$$

It is seen that Equation (2.1) has infinitely many solutions (Figure 1).

Suppose $\left\{y_{n}\right\}_{n=-\infty, n \neq 0}^{n=\infty}$ are solutions of Equation 2.1), where $y_{-n} \in\left[-\left(2 n+\frac{1}{2}\right) \pi,-\left(2 n+\frac{1}{4}\right) \pi\right)$ and $y_{n} \in$ $\left[\left(2 n+\frac{1}{4}\right) \pi,\left(2 n+\frac{1}{2}\right) \pi\right), n=1,2,3, \ldots$ Now, from Equation 2.2$), x_{n}=1-y_{n} \cot y_{n}$ for $n= \pm 1, \pm 2, \pm 3, \ldots$ Consequently, It gives that $g_{\lambda}^{\prime}\left(z_{n}\right)=0$ so $z_{n}=x_{n}+i y_{n}$ are critical points for $g_{\lambda}(z)$. The critical values of $g_{\lambda}(z)$ are given by $g_{\lambda}\left(z_{n}\right)$. It is observed that $g_{\lambda}\left(z_{n}\right)$ are distinct for different $n$. It shows that the function $g_{\lambda}(z)$ has infinitely many critical values.

Since $g_{\lambda}(z)$ tends to 0 as $z$ tends to infinity along positive real axis, it gives that the finite asymptotic value of $g_{\lambda}(z)$ is 0 .

Thus, it follows that the function $g_{\lambda} \in \mathcal{G}$ possesses infinitely many singular values. 


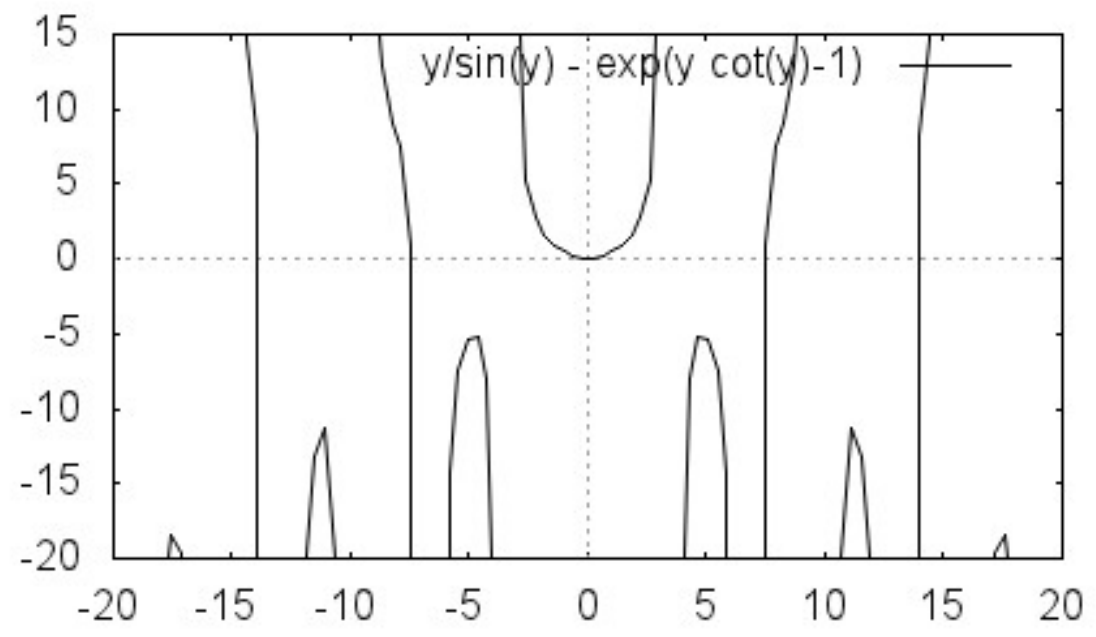

Figure 1: Graph of $\frac{y}{\sin y}-e^{y \cot y-1}$

The following theorem explains the function $g_{\lambda}(z)$ has no critical points in the right half plane:

Theorem 2.2. Let $g_{\lambda} \in \mathcal{G}$. Then, the function $g_{\lambda}^{\prime}(z)$ has no zeros in the right half plane $H^{+}=\{z \in \hat{\mathbb{C}}:$ $\operatorname{Re}(z)>0\}$.

Proof. Suppose $\operatorname{Re}(z)>0$, and $g_{\lambda}^{\prime}(z)=\lambda \frac{(1-z) e^{z}-1}{\left(e^{z}-1\right)^{2}}=0$ which implies that $e^{-z}=1-z$. Then,

$$
\frac{\cos y-i \sin y}{e^{x}}=1-x-i y
$$

Firstly, when $y \neq 0$, then, by imaginary part of Equation $2.3, \frac{\sin y}{y}=e^{x}>1$. This is not true for $y>0$ and for $y<0$ because $\frac{\sin y}{y}$ is an even function.

Secondly, when $y=0$, then $z=x>0$ and, by real part of Equation $\sqrt{2.3}, e^{x}=\frac{1}{1-x}$. For $x>1$, it is not valid because the left hand side is positive and the right hand side is negative. It is obviously not true when $x=1$. This is also false for $0<x<1$. Consequently, the function $g_{\lambda}^{\prime}(z)$ has no zeros in the right half plane $H^{+}$.

In the following theorem, it is shown that the function $g_{\lambda}(z)$ maps the left half plane outside the open disk centered at origin and having radius $\lambda$.

Theorem 2.3. Let $g_{\lambda} \in \mathcal{G}$. Then, the function $g_{\lambda}(z)$ maps the left half plane $H^{-}=\{z \in \hat{\mathbb{C}}: \operatorname{Re}(z)<0\}$ outside the open disk centered at origin and having radius $\lambda$.

Proof. Consider the line segment $\gamma$ is defined by $\gamma(t)=t z, t \in[0,1]$ and the function $h(z)=e^{z}$ for an arbitrary fixed $z \in H^{-}$. Then

$$
\int_{\gamma} h(z) d z=\int_{0}^{1} h(\gamma(t)) \gamma^{\prime}(t) d t=z \int_{0}^{1} e^{t z} d t=e^{z}-1
$$

Since $M \equiv \max _{t \in[0,1]}|h(\gamma(t))|=\max _{t \in[0,1]}\left|e^{t z}\right|<1$ for $z \in H^{-}$and

$$
\left|e^{z}-1\right|=\left|\int_{\gamma} h(z) d z\right| \leq M|z|<|z|
$$

so $\left|\frac{z}{e^{z}-1}\right|>1$ for all $z \in H^{-}$. Therefore, it follows that $\left|g_{\lambda}(z)\right|=\left|\lambda \frac{z}{e^{z}-1}\right|>|\lambda|$ for all $z \in H^{-}$. This shows that the function $g_{\lambda}(z)$ maps the left half plane $H^{-}$outside the open disk centered at origin and having radius $\lambda$. 
Remark 2.4. For the zeros of $g_{\lambda}^{\prime}(z)$ on imaginary axis, from Equation $(2.3)$, it is found that $\cos y-i \sin y=$ $1-i y$. This equation gives $y=0$. Therefore, $g_{\lambda}^{\prime}(z)$ has no zeros on imaginary axis.

The following theorem proves that the function $g_{\lambda}(z)$ has all the critical values outside the open disk centered at origin and having radius $\lambda$ :

Theorem 2.5. Let $g_{\lambda} \in \mathcal{G}$. Then, all the critical values of $g_{\lambda}(z)$ lie outside the open disk centered at origin and having radius $\lambda$.

Proof. By Theorem 2.2, the function $g_{\lambda}^{\prime}(z)$ has no zeros in the right half plane $H^{+}$. Consequently, all the critical points lie in the left half plane $H^{-}$. But, by using Theorem 2.3 , the function $g_{\lambda}(z)$ maps the left half plane $H^{-}$outside the open disk centered at origin and having radius $\lambda$. Hence, it proves that all the critical values of $g_{\lambda}(z)$ lie outside the open disk centered at origin and having radius $\lambda$.

\section{Nature of Real Fixed Points of $g_{\lambda} \in \mathcal{G}$}

In the present section, the existence of real fixed points of the function $g_{\lambda}(x)=\lambda g(x)$, where $g(x)=\frac{x}{e^{x}-1}$, and their nature are studied. For sake of convenience, consider the function $\phi(x)=x g^{\prime}(x)+g(x)$ for $x \geq 0$. It means that

$$
\phi(x)=x \frac{\left(e^{x}-1-x e^{x}\right)}{\left(e^{x}-1\right)^{2}}+\frac{x}{\left(e^{x}-1\right)}=\frac{x}{\left(e^{x}-1\right)^{2}}\left[(2-x) e^{x}-2\right]
$$

If $q(x)=(2-x) e^{x}-2$, then $q^{\prime}(x)=(1-x) e^{x}$ and $q^{\prime \prime}(x)=-x e^{x}$. It is seen that $q^{\prime \prime}(x)<0$ for $x \in \mathbb{R}^{+}$. Therefore, the function $q^{\prime}(x)$ is decreasing on $\mathbb{R}^{+}$. Since $q^{\prime}(0)=1$ and $q^{\prime}(x) \rightarrow-\infty$ as $x \rightarrow+\infty$, by continuity of $q^{\prime}(x)$, it follows that there is a unique $\tilde{x}>0$ such that $q^{\prime}(x)>0$ for $0 \leq x<\tilde{x}, q^{\prime}(\tilde{x})=0$ and $q^{\prime}(x)<0$ for $x>\tilde{x}$. Thus, $q(x)$ increases in $[0, \tilde{x})$, attains its maximum at $\tilde{x}$ and decreases thereafter. It ensures from the facts $q(0)=0$ and $q(x) \rightarrow-\infty$ as $x \rightarrow+\infty$ that there is a unique positive $x^{*}>\tilde{x}$ such that $q(x)>0$ for $0<x<x^{*}, q\left(x^{*}\right)=0$ and $q(x)<0$ for $x>x^{*}$. Since $\frac{x}{\left(e^{x}-1\right)^{2}}>0$ for all $x>0$. We have

$$
\phi(x)=\frac{x}{\left(e^{x}-1\right)^{2}} q(x) \begin{cases}>0 & \text { for } 0<x<x^{*} \\ =0 & \text { for } x=x^{*} \\ <0 & \text { for } x>x^{*}\end{cases}
$$

Let us define

$$
\lambda^{*}=\frac{x^{*}}{g\left(x^{*}\right)}=e^{x^{*}}-1
$$

where $x^{*}$ is the unique positive real root of the equation $(2-x) e^{x}-2=0$.

The following theorem shows that the function $g_{\lambda}(x)$ has a unique real fixed point:

Theorem 3.1. Let $g_{\lambda} \in \mathcal{G}$. Then, the function $g_{\lambda}(x)$ has a unique real fixed point $x_{\lambda}$.

Proof. Since $g_{\lambda}(x)>0$ for all $x \in \mathbb{R}$, each real fixed point of $g_{\lambda} \in \mathcal{G}$ is positive. The function $g_{\lambda}^{\prime}(x)=$ $\lambda \frac{(1-x) e^{x}-1}{\left(e^{x}-1\right)^{2}}<0$ for $x>0$ and hence $g_{\lambda}(x)$ is decreasing on $\mathbb{R}^{+}$.

Let $h_{\lambda}(x)=g_{\lambda}(x)-x$ for $x \in \mathbb{R}$. Since $g_{\lambda}^{\prime}(x)<0$ for $x>0$ and $h_{\lambda}^{\prime}(x)=g_{\lambda}^{\prime}(x)-1<0$, then the function $h_{\lambda}(x)$ is decreasing on $\mathbb{R}^{+}$. Now, $h_{\lambda}(0)=\lambda>0, h_{\lambda}(x) \rightarrow-\infty$ as $x \rightarrow+\infty$ and $h_{\lambda}(x)$ is continuous on $\mathbb{R}^{+}$. By the intermediate value theorem, there exists a unique positive $x_{\lambda}$ such that $h_{\lambda}\left(x_{\lambda}\right)=0$. It proves that $g_{\lambda}(x)$ has a unique positive fixed point $x_{\lambda}$.

In the following theorem, the nature of fixed points of $g_{\lambda}(x)$ are determined:

Theorem 3.2. Let $g_{\lambda} \in \mathcal{G}$. Then, the fixed point $x_{\lambda}$ of the function $g_{\lambda}(x)$ is (i) attracting for $0<\lambda<\lambda^{*}$ (ii) rationally indifferent for $\lambda=\lambda^{*}$ (iii) repelling for $\lambda>\lambda^{*}$. 
Proof. Since the derivative of $\frac{x}{g(x)}$ is positive for $x>0$, the function $\frac{x}{g(x)}$ is increasing on $\mathbb{R}^{+}$. Using this fact, we prove the following cases for different values of parameter $\lambda$ :

(i) For $0<\lambda<\lambda^{*}$, since the function $\frac{x}{g(x)}$ is increasing on $\mathbb{R}^{+}$and $\lambda=\frac{x_{\lambda}}{g\left(x_{\lambda}\right)}$, we have $\frac{x_{\lambda}}{g\left(x_{\lambda}\right)}<\frac{x^{*}}{g\left(x^{*}\right)}$. It means that $e^{x_{\lambda}}<e^{x^{*}}$. Hence, $x_{\lambda}<x^{*}$. By Equation 3.1 , $\phi\left(x_{\lambda}\right)>0$. Since $g_{\lambda}^{\prime}\left(x_{\lambda}\right)=\frac{\phi\left(x_{\lambda}\right)}{g\left(x_{\lambda}\right)}-1$, it follows that $g_{\lambda}^{\prime}\left(x_{\lambda}\right)+1=\frac{\phi\left(x_{\lambda}\right)}{g\left(x_{\lambda}\right)}>0$. Since $g_{\lambda}^{\prime}(x)$ is negative on $\mathbb{R}^{+}$, it shows that $-1<g_{\lambda}^{\prime}\left(x_{\lambda}\right)<0$ and consequently, the fixed point $x_{\lambda}$ of $g_{\lambda}(x)$ is an attracting for $0<\lambda<\lambda^{*}$.

(ii) For $\lambda=\lambda^{*}$, it is easy to prove $x_{\lambda}=x^{*}$. Now, by Equation 3.1 , it follows that $g_{\lambda}^{\prime}\left(x_{\lambda}\right)+1=\frac{\phi\left(x_{\lambda}\right)}{g\left(x_{\lambda}\right)}=0$ which implying $g_{\lambda^{*}}^{\prime}\left(x_{\lambda}\right)=-1$. Therefore, the fixed point $x^{*}$ of $g_{\lambda}(x)$ is rationally indifferent for $\lambda=\lambda^{*}$.

(iii) For $\lambda>\lambda^{*}$, by similar arguments used in (i), it follows that $x_{\lambda}>x^{*}$. Again, by Equation (3.1) and by the fact $x_{\lambda}>x^{*}$, we have $\phi\left(x_{\lambda}\right)<0$. It gives that $g_{\lambda}^{\prime}\left(x_{\lambda}\right)+1=\frac{\phi\left(x_{\lambda}\right)}{g\left(x_{\lambda}\right)}<0$ and hence $g_{\lambda}^{\prime}\left(x_{\lambda}\right)<-1$. Therefore, $x_{\lambda}$ is a repelling fixed point of $g\left(x_{\lambda}\right)$ for $\lambda>\lambda^{*}$.

It is observed from Theorem 3.2 that the nature of the fixed point changes whenever parameter $\lambda$ crosses parameter value $\lambda^{*}$.

Remark 3.3. For $\lambda>\lambda^{*}$, there may exist periodic points of period greater than or equal to 2 . This case is not considered in this paper. It is left for forthcoming work.

The comparisons among the analogous results of $g_{\lambda}(z)=\lambda \frac{z}{e^{z}-1}, T_{\lambda}(z)=\lambda \tan z[2], E_{\lambda}(z)=\lambda \frac{e^{z}-1}{z}$ [5] and $f_{\lambda}(z)=\lambda \frac{z}{z+4} e^{z}[12$ for $\lambda>0$ are shown in Table 1 .

Table 1: Comparisons among the analogous results of the families of functions

\begin{tabular}{|c|c|c|c|}
\hline$g_{\lambda}(z)=\lambda \frac{z}{e^{z}-1}$ & $T_{\lambda}(z)=\lambda \tan z$ & $E_{\lambda}(z)=\lambda \frac{e^{z}-1}{z}$ & $f_{\lambda}(z)=\lambda \frac{z}{z+4} e^{z}$ \\
\hline $\begin{array}{l}g_{\lambda}(z) \text { is transcenden- } \\
\text { tal meromorphic func- } \\
\text { tion. } \\
g_{\lambda}(z) \text { has infinitely } \\
\text { many poles. } \\
g_{\lambda}(z) \text { has transcen- } \\
\text { dental meromorphic } \\
\text { Schwarzian Deriva- } \\
\text { tive. } \\
g_{\lambda}(z) \text { is neither even } \\
\text { nor odd function. } \\
g_{\lambda}(z) \text { has infinitely } \\
\text { many critical values. } \\
g_{\lambda}(z) \text { has only one fi- } \\
\text { nite asymptotic value } \\
0 \text {. All singular values } \\
\text { of } g_{\lambda}(z) \text { are outside } \\
\text { of open disk except } \\
\text { asymptotic value } 0 .\end{array}$ & $\begin{array}{l}T_{\lambda}(z) \text { is transcenden- } \\
\text { tal meromorphic func- } \\
\text { tion. } \\
T_{\lambda}(z) \text { has infinitely } \\
\text { many poles. } \\
T_{\lambda}(z) \text { has polynomial } \\
\text { Schwarzian Deriva- } \\
\text { tive. } \\
T_{\lambda}(z) \text { is an odd func- } \\
\text { tion. } \\
T_{\lambda}(z) \text { has no critical } \\
\text { values. } \\
T_{\lambda}(z) \text { has two finite } \\
\text { asymptotic values } \\
\pm \lambda i \text {. } \\
\text { All singular values of } \\
T_{\lambda}(z) \text { are bounded. }\end{array}$ & $\begin{array}{l}E_{\lambda}(z) \text { has no pole. } \\
E_{\lambda}(z) \text { has transcen- } \\
\text { dental meromorphic } \\
\text { Schwarzian Deriva- } \\
\text { tive. } \\
E_{\lambda}(z) \text { is neither even } \\
\text { nor odd function. } \\
E_{\lambda}(z) \text { has infinitely } \\
\text { many critical values. } \\
E_{\lambda}(z) \text { has only one fi- } \\
\text { nite asymptotic value } \\
0 . \\
\text { All singular values of } \\
E_{\lambda}(z) \text { are bounded. }\end{array}$ & $\begin{array}{l}f_{\lambda}(z) \text { is transcenden- } \\
\text { tal meromorphic func- } \\
\text { tion. } \\
f_{\lambda}(z) \text { has only one } \\
\text { pole. } \\
f_{\lambda}(z) \text { has rational } \\
\text { Schwarzian Deriva- } \\
\text { tive. } \\
f_{\lambda}(z) \text { is neither even } \\
\text { nor odd function. } \\
f_{\lambda}(z) \text { has finitely } \\
\text { many critical values. } \\
f_{\lambda}(z) \text { has only one fi- } \\
\text { nite asymptotic value } \\
0 . \\
\text { All singular values of } \\
f_{\lambda}(z) \text { are bounded. }\end{array}$ \\
\hline
\end{tabular}

\section{References}

[1] A. F. Beardon, Iteration of Rational Functions, Springer, New York, (1991). 1 
[2] R. L. Devaney, L. Keen, Dynamics of meromorphic maps: Maps with polynomial Schwarzian derivative, Ann. Sci. Ec. Norm. Sup., 22 (1989), 55-79. 3

[3] X. H. Hua, C. C. Yang, Dynamics of Transcendental Functions, Gordon and Breach Sci. Pub., Amsterdam, (1998). 1

[4] K. Ireland, M. Rosen, A Classical Introduction to Modern Number Theory, 2nd Ed., Springer-Verlag, New York, (1998). 1

[5] G. P. Kapoor, M. Guru Prem Prasad, Dynamics of $\left(e^{z}-1\right) / z$ : the Julia set and Bifurcation, Ergod. Th. Dynam. Sys., 18 (1998), 1363-1383. 1 , 3

[6] S. Morosawa, Y. Nishimura, M. Taniguchi, T. Ueda, Holomorphic Dynamics, Cambridge University Press, Cambridge, (2000). 1

[7] T. Nayak, M. Guru Prem Prasad, Iteration of certain meromorphic functions with unbounded singular values, Ergod. Th. Dynam. Sys., 30 (2010), 877-891. 1

[8] M. Guru Prem Prasad, Chaotic burst in the dynamics of $f_{\lambda}(z)=\lambda \frac{\sinh (z)}{z}$, Regul. Chaotic Dyn., 10 (2005), 71-80. 1

[9] M. Guru Prem Prasad, Tarakanta Nayak, Dynamics of certain class of critically bounded entire transcendental functions, J. Math. Anal. Appl. 329 (2007), 1446-1459. 1

[10] G. Rottenfusser, J. Ruckert, L. Rempe, D. Schleicher, Dynamics rays of bounded-type entire functions, Ann. Math., 173 (2011), 77-125. 1

[11] M. Sajid, G. P. Kapoor, Dynamics of a family of non-critically finite even transcendental meromorphic functions, Regul Chaotic Dyn., 9 (2004), 143-162. 1

[12] M. Sajid, Real and complex dynamics of one parameter family of meromorphic functions, Far East J. Dynamical Sys., 19 (2012), 89-105. 3

[13] J. Zheng, Singular Values of Meromorphic Functions, Value Distribution of Meromorphic Functions, Springer, (2011), 229-266. 1 